\title{
Comparação de Diferentes Estratégias para a Análise de Características de Crescimento e de Carcaça de Suínos Cruzados: Modelos Finito e Infinitesimal Poligênico ${ }^{1}$
}

\author{
Tarcísio de Moraes Gonçalves ${ }^{2}$, Henrique Nunes de Oliveira ${ }^{3}$, Henk Bovenhuis ${ }^{4}$, Marco Bink ${ }^{4}$, \\ Johan Van Arendonk ${ }^{4}$
}

\begin{abstract}
RESUMO - Foi utilizada uma análise de segregação com o uso da inferência Bayesiana para estimar componentes de variância e verificar a presença de genes de efeito principal (GEP) influenciando duas características de carcaça: gordura intramuscular (GIM), em \%, e espessura de toucinho (ET), em mm; e uma de crescimento, ganho de peso (g/dia) dos 25 aos 90 kg de peso vivo (GP). Para este estudo, foram utilizadas informações de 1.257 animais provenientes de um delineamento de $\mathrm{F}_{2}$, obtidos do cruzamento de suínos machos Meishan e fêmeas Large White e Landrace. No melhoramento genético animal, os modelos poligênicos finitos (MPF) podem ser uma alternativa aos modelos poligênicos infinitesimais (MPI) para avaliação genética de características quantitativas usando pedigrees complexos. MPI, MPF e MPI combinado com MPF foram empiricamente testados para se estimar componentes de variâncias e número de genes no MPF. Para a estimação de médias marginais a posteriori de componentes de variância e de parâmetros, foi utilizada uma metodologia Bayesiana, por meio do uso da Cadeia de Markov, algoritmos de Monte Carlo (MCMC), via Amostrador de Gibbs e Reversible Jump Sampler (Metropolis-Hastings). Em função dos resultados obtidos, pode-se evidenciar quatro GEP, sendo dois para GIM e dois para ET. Para ET, o GEP explicou a maior parte da variação genética, enquanto, para GIM, o GEP reduziu significativamente a variação poligênica. Para a variação do GP, não foi possível determinar a influência do GEP. As herdabilidades estimadas ajustandose MPI para GIM, ET e GP foram de 0,37; 0,24 e 0,37, respectivamente. Estudos futuros com base neste experimento que usem marcadores moleculares para mapear os genes de efeito principal que afetem, principalmente GIM e ET, poderão lograr êxito.
\end{abstract}

Palavras-chave: espessura de toucinho, ganho de peso, gordura intramuscular, inferência Bayesiana, modelos finito e infinitesimal

\section{Comparison of Different Strategies to Analyze Growth and Carcass Traits in a Crossbred Pig Population: Finite and Infinitesimal Polygenic Models}

\begin{abstract}
A Bayesian marker-free segregation analysis was applied for the estimation of variance components and to search for evidence of segregation genes affecting two carcass traits: intramuscular fat (IMF), \%, and backfat thickness (BF), mm ; and one growth trait: body weight gain (LG) from 25 to $90 \mathrm{~kg}$, approximately, g/day. In this study, 1,257 animals from the F2 design produced by breeding among pigs Meishan (male) and Dutch Large White and Landrace lines (female) were used. In animal breeding, finite polygenic models (FPM) may be an alternative to the infinitesimal polygenic model (IPM) for genetic evaluation of populations with multiple-generations pedigree for quantitative traits. FPM, IPM and FPM combined with IPM were empirically tested for estimation of variance components and number of genes in FPM. Estimation of marginal posteriori means of variance components and parameters were performed by using Markov Chain Monte Carlo techniques with the Gibbs sampler and the reversible Jump sampler (Metropolis-Hastings). The results showed evidence for four Major Genes (MG), i.e., two for IMF and two BF. For BF, the MG explained almost all of genetic variance, while for IMF, MG reduced the polygenic variance significantly. The LG was not likely influenced by MG. The polygenic heritability estimates for IMF, BF and LG were 0.37, 0.24 and 0.37, respectively. Further molecular genetic research, based on the same experimental data, aiming to map the major genes estimated for IMF and BF has a high probability of success.
\end{abstract}

Key Words: backfat thickness, bayesian inference, finite and infinitesimal models, intramuscular fat, body weight gain

\section{Introdução}

Muitas características são afetadas por um ou mais (GEP) e por poligenes em algumas espécies (Falconer \& Mackay, 1996). Nestes casos, a separação dos efeitos e a identificação de genes individuais que expliquem a variação fenotípica são cruciais para se entender o mecanismo básico da arquitetura genética de características quantitativas e, desta forma, fornecer informações sobre o delineamento de estratégias ótimas para os programas de melhoramento de plantas e de animais. Como a herança destas características influenciadas por poucos genes é diferente das características quantitativas tradicionais, os mé-

\footnotetext{
1 Projeto financiado pelo CNPq

2 Prof. Adjunto do DZO da UFLA - CP 37 - 37200-000 - Lavras - MG. E.mail: tarcisio@ufla.br

3 Prof. Associado da UNESP - Botucatu. E.mail: hnunes@fca.unesp.br

4 Prof. da Wageningen University, Animal Sciences, Animal Breeding and Genetics Group, Wageningen, Holanda.
} 
todos clássicos de análise não são apropriados para estes estudos. Todavia, a genética quantitativa, baseada em novas tecnologias estatísticas e computacionais, avançou ao ponto em que se pode descobrir genes individuais e suas localizações nos cromossomos (Hoeschele et al., 1997; Bink et al., 2002a).

Nosúltimos dez anos, metodologias estatísticas para a detecção de GEP, baseadas em informações de genealogia e fenótipo, têm sido desenvolvidas para populações de animais. Esses métodos consistem no uso de modelos mistos e de análise de segregação para ajustar os dados à uma mistura de modelos genéticos que inclui, além dos efeitos poligênicos, o efeito bialélico do GEP. Por meio desses métodos, foram descobertos GEP para características de carcaça em suínos a partir de uma mistura de modelo genético e amostrador de Gibbs (Janss et al., 1995, 1997).

Quase todas as estimativas de componentes de variâncias aditiva e não-aditiva encontradas na literatura foram obtidas pelo modelo poligênico infinitesimal (MPI) por meio do método de estimação da máxima verossimilhança (Hoeschele, 1991; Fuerst \& Soelkner, 1994) ou com os métodos não-iterativos mais simples (Allaire \& Henderson, 1965; Hay et al., 1983). O modelo poligênico finito (MPF) foi proposto pela primeira vez por Thompson \& Skolnick (1977) para estimativa da herdabilidade da longevidade, utilizando-se informações de pedigrees mais complexos em humanos, e, posteriormente, por Fernando et al. (1994), para análise de segregação complexa. Para esses MPF iniciais, presumiu-se loco bialélico, ação aditiva dos genes, constância dos efeitos aditivos e das freqüências dos alelos por meio dos locos. Além disso, ajustou-se um efeito poligênico em lugar de genótipos de locos individuais. Recentemente, MPF foi explorado como um modo alternativo para se calcularem os componentes de variância genéticos (Du et al., 1999; Pong-Wong et al., 1998, 1999).

Realizou-se este trabalho para examinar modelos alternativos para se estimar componentes de variância e identificar GEP, em delineamento de animais $\mathrm{F}_{2}$, obtidos do cruzamento de suínos machos da raça chinesa Meishan e fêmeas das linhagens holandesas Large White e Landrace, com base em metodologia Bayesiana, com o uso da Cadeia de Markov, algoritmos de Monte Carlo (MCMC). Esta metodologia tem vários atrativos teóricos, como a habilidade para extrair mais informações dos dados que as outras metodologias e menor propensão a vícios no estudo de populações selecionadas (Hoeschele et al., 1997).

\section{Material e Métodos}

Foram coletadas informações das características gordura intramuscular (GIM), em \%, espessura de toucinho (ET), em mm, obtidas por ultra-som, e ganho de peso dos 25 aos $90 \mathrm{~kg}$ (GP), em g/dia, das gerações $\mathrm{F}_{1}$ (298 animais) e $\mathrm{F}_{2}$ (831 animais) de suínos. O nível de gordura intramuscular (GIM) foi determinado 24 horas após o abate dos animais, em uma amostra do músculo Longissimus dorsi (Hovenie et al., 1993).

Estas progênies originaram-se do experimento descrito por Janss et al. (1997), que envolveu cinco companhias de melhoramento animal, no qual foram cruzados machos da raça Meishan (18 animais) com fêmeas das linhagens holandesas Large White e Landrace (110 animais).

Na Tabela 1 são apresentados os números de observações, as médias e os respectivos desviospadrão para as características estudadas.

Os modelos lineares mistos poligênicos utilizados para descrever as observações, em termos matriciais, foram:

a) Modelo poligênico infinitesimal (MPI) combinado com modelo poligênico finito (MPF):

$$
\underset{\sim}{y}=X \underset{\sim}{b}+\underset{\sim}{W}+\sum_{K}^{N_{G E P}} Z_{G E P} \alpha_{G E P, K}+\underset{\sim}{e}
$$

em que $y$ é o vetor das observações; $X$, a matriz de incidência dos efeitos não-genéticos; $\underset{\sim}{b}$, o vetor de efeitos não-genéticos: companhia de melhoramento (5), sexo (macho e fêmea), dia de abate NID (0, $\left.\sigma_{\mathrm{da}}^{2}\right)$, classes de peso ao abate $\operatorname{NID}\left(0, \sigma_{\mathrm{pa}}^{2}\right)$, classes de peso vivo NID $\left(0, \sigma_{p v}^{2}\right) ; \mathrm{W}$, a matriz de incidência dos efeitos aleatórios genéticos diretos; $\underset{\sim}{u}$, o vetor dos efeitos aleatórios dos valores genéticos diretos do animal, NID $\left(0, A \sigma^{2}{ }_{\mathrm{u}}\right) ; \mathrm{Z}_{\mathrm{GEP}}$ é tipicamente não conhecida, uma vez que os genótipos dos indivíduos não são conhecidos. Entretanto, esta matriz pode ser inferida a partir do pedigree e do fenótipo; $\mathrm{N}_{\mathrm{GEP}}$ é o número de (GEP), tendo sido considerado como um, tornando possível a comparação com as análises efetuadas por Janss et al. (1997); $\alpha_{G E P, K}$, um vetor bidimensional $\left[\mathrm{a}_{\mathrm{K}} \mathrm{d}_{\mathrm{K}}\right]^{\text {' }}$ para o késimo loco de um GEP, isto é, onde são ajustados os efeitos aditivo (a) e de dominância (d), assumindo-se que os locos para GEP são bialélicos; $\stackrel{e}{,}$, o vetor dos erros associados a cada observação, $\operatorname{NID}\left(0, \sigma^{2}\right)$. 
Tabela 1 - Número de observações (N), médias e respectivos desvios-padrão (DP) para as características estudadas na geração $\mathrm{F}_{2}$

Table 1 - Number of observations, averages and standard deviations (DP) for the traits studied in the $F_{2}$ generation

\begin{tabular}{|c|c|c|c|}
\hline $\begin{array}{l}\text { Característica } \\
\text { Trait }\end{array}$ & $\mathrm{N}$ & $\begin{array}{l}\text { Média } \\
\text { Average }\end{array}$ & DP \\
\hline $\begin{array}{l}\text { Gordura intramuscular (\%) } \\
\text { GIM intramuscular fat (\%) }\end{array}$ & 831 & 1,84 & 0,87 \\
\hline $\begin{array}{l}\text { Espessura de toucinho (mm) de animais com aproximadamente } 90 \mathrm{~kg} \\
\text { Backfat thickness }(\mathrm{mm}) \text { of animals with approximately } 90 \mathrm{~kg}\end{array}$ & 805 & 15,33 & 3,65 \\
\hline $\begin{array}{l}\text { Ganho de peso de aproximadamente } 25 \text { a } 90 \mathrm{~kg} \text { (g/dia) } \\
\text { Weight gain with approximately } 25 \text { to } 90 \mathrm{~kg} \text { (g/days) }\end{array}$ & 745 & 656,19 & 128,77 \\
\hline
\end{tabular}

b) Modelo poligênico infinitesimal (MPI):

$$
\underset{\sim}{y}=X \underset{\sim}{b}+W \underset{\sim}{u}+\underset{\sim}{e}
$$

Assumiu-se efeito poligênico, com grande número de locos e nenhum (GEP), em que os efeitos são os mesmos, como definidos no modelo (1).

\section{c) Modelo poligênico finito (MPF):}

Para se ajustar um número finito de locos poligênicos (cinco), não ligados a marcadores moleculares, os quais descrevem a covariância genética entre os membros do pedigree, a estimativa dos componentes de variância foi feita utilizando-se o modelo:

$$
\underset{\sim}{y}=X \underset{\sim}{b}+\sum_{K}^{N_{G E P}} Z_{G E P} \alpha_{G E P, K}+\underset{\sim}{e}
$$

em que todos os efeitos são definidos anteriormente no modelo (1).

Na aplicação desses modelos, foram considerados os efeitos não-genéticos dia do abate, companhia de melhoramento, sexo e 10 classes de peso ao abate para GP e GIM. Para ET, foram consideradas nove classes de peso vivo. De forma similar à adotada por Sillanpää \& Arjas (1998) e Bink et al. (2002b), as seguintes distribuições $a$ priori foram adotadas como sugestão: distribuição uniforme $(0,1)$, para a média, o sexo e a companhia de melhoramento, e normal e independentemente distribuída, com média zero e variância desconhecida, para dia do abate e as classes de peso consideradas nas análises. A distribuição $a$ priori assumida para os efeitos genéticos foi NID (0, $\mathrm{A} \sigma^{2}{ }_{\mathrm{u}}$ ), sendo $\mathrm{A}$ a matriz dos coeficientes de parentes- co entre os animais e, para $\sigma^{2}{ }_{\mathrm{u}}, \sigma^{2}{ }_{\mathrm{e}}, \sigma_{\mathrm{da}}^{2}, \sigma_{\mathrm{pa}}^{2}, \sigma_{\mathrm{py}}^{2}$, foi presumida a distribuição de qui-quadrado $\left(\chi^{2}\right)$ escalonada invertida. O número de GEP ( $\left.\mathrm{N}_{\mathrm{GEP}}\right)$ foi considerado variável aleatória e sua distribuição foi inferida a partir dos dados ("Reversible Jump Sampler - Metropolis-Hastings” descrito por Green, 1995). Presumiu-se a distribuição de Poisson (truncada) com média l (com um máximo pré-definido [ $\mathrm{N}_{\mathrm{GEP}}=1$ ou 5 ], para os modelos 1 e 3, respectivamente). Para um provável GEP, presumiu-se que a distribuição a priori para os efeitos aditivos e de dominância era a uniforme e, para as freqüências alélicas, foi assumida a função Beta.

Os genótipos para um loco foram determinados conjuntamente por meio dos alelos da população fundadora (com pais desconhecidos) e por indicadores de segregação de indivíduos não-fundadores (com pais conhecidos), com os locos da população fundadora em equilíbrio de ligação e Hardy - Weinberg (Lange \& Matthysse, 1989; Thompson, 1994; Sobel \& Lange, 1996). Os indicadores de segregação descrevem unicamente o fluxo gênico por meio do pedigree e a implementação foi similar à descrita por Uimari \& Sillanpää (2001) e Bink et al. (2002a) para o mapeamento de QTLs.

Para a distribuição condicional das observações (Y), foi assumida a Normal e, quando combinada com as distribuições $a$ priori, foi obtida a distribuição $a$ posteriori conjunta de todos os parâmetros de interesse. Uma vez que esta distribuição não possui forma padrão conhecida, amostras marginais desta distribuição foram geradas pelo método de Monte Carlo via Cadeia de Markov (MCMC). Na simulação MCMC, o amostrador de Gibbs foi utilizado para se obterem amostras das distribuições marginais das variáveis aleatórias. $\mathrm{O} \mathrm{N}_{\mathrm{GEP}}$ foi incluído nos modelos 1 e 3 como uma variável adicional não conhecida por meio do 
Foram gerados 500 mil ciclos do amostrador de Gibbs. Em cada análise MCMC, considerando-se uma única cadeia de Markov, o período de descarte amostral adotado (burn-in) foi de mil ciclos (amostras). O intervalo de utilização amostral foi de 50 ciclos, visto que as amostras consecutivas são altamente correlacionadas. $\mathrm{O}$ total de amostras gerado foi de 10 mil, sendo, portanto, aproveitadas para análise 9 mil amostras das distribuições a posteriori dos parâmetros.

Para o uso desta metodologia, foi empregado o pacote computacional FlexQTL ${ }^{\mathrm{TM}}$, desenvolvido por Bink et al. (2002a), pesquisador da Universidade de Wageningen, a partir dos estudos descritos por Uimari \& Sillanpää (2001).

\section{Resultados e Discussão}

As estimativas das médias e os respectivos desvios-padrão dos componentes de variância e de herdabilidade $\left(\mathrm{h}^{2}\right)$ para as características estudadas são apresentados na Tabela 2. As inferências sobre as distribuições a posteriori das $\mathrm{h}^{2}$ foram obtidas com base nas amostras de Gibbs dos componentes de variância e utilizando-se estes valores para sumarizar as distribuições a posteriori das $\mathrm{h}^{2}$. As estimativas das variâncias poligênicas sugerem a presença de quantidade razoável de variação genética. Estas estimativas correspondem à herdabilidade poligênica, sem considerar as variações causadas por GEP. Os valores variaram de 0,24 a 0,37 e são similares aos obtidos em estudos anteriores com a mesma base de dados, porém, com metodologia de análise diferente (Janss et al., 1997; De Koning et al., 2001; Szida et al., 2002). Entretanto, são inferiores à $\mathrm{h}^{2}$ de 0,61 para ET e 0,52 para GIM (Warris et al., 1990), e de 0,51 para ET e 0,61 para GIM (Hovenier et al., 1993) que foram estimadas a partir da base de dados e metodologia de análise diferente.

Na Tabela 2 são apresentadas as estimativas marginais médias a posteriori para as variâncias genéticas aditiva, de dominância, residual e herdabilidades com seus respectivos desvios-padrão, assumindo-se cinco locos poligênicos. As variâncias genéticas explicadas pelo GEP foram diferentes nos modelos quando se ajustou ou não o efeito de dominância e foram quase o dobro da variância poligênica estimada no MPI. Isso pode ser explicado pela segregação de cinco ou menos GEP (Tabelas 3 e 4) que causam variações, atribuídas à variância residual no MPI, mas que podem ser incluídas como variância genética no MPF. Para todas as características, as estimativas médias a posteriori da variância genética aditiva e, conseqüentemente, de $\mathrm{h}^{2}$ foram afetadas pela inclusão ou não da variância da dominância na análise. As estimativas médias das variâncias genéticas aditivas, sem considerar a dominância, foram superiores às das variâncias genéticas poligênicas no MPI (Tabela 2; Figura 1).

As $\mathrm{h}^{2}$ estimadas nos modelos considerando-se somente os efeitos aditivos foram diferentes e superiores às do MPI e a variância residual foi menor (Tabela 2). Quando estimada a variância da dominância no modelo, o mesmo ocorreu com as $\mathrm{h}^{2}$ para as características GIM e ET, mas não com a variável GP (Tabela 2; Figura 2). O número esperado de locos para se obter a média a posteriori da variância da dominância (Tabelas 3 e 4) variou, necessitando de mais um loco (GEP), o que não ocorreu com a característica GP [P( $\left.\mathrm{N}_{\mathrm{GEP}}\right)$ $=0,8848]$, sugerindo dependência dos componentes de variância a serem estimados em relação ao número de locos na análise utilizando-se o MPF. As estimativas das variâncias genéticas aditiva e de dominância adotando-se MPF variaram em função de se considerar o efeito de dominância no modelo (Tabela 4). A característica ET foi a que menos sofreu alteração dos valores das variâncias genética aditiva.

Ao serem combinados os MPI e MPF, as estimativas médias a posteriori das variâncias residuais e genéticas para a característica GIM (Tabela 2) foram semelhantes aos valores apresentados por Janss et al. (1997). Ao se considerar o efeito da dominância no modelo, observou-se que GIM e ET foram influenciados significativamente por um único gene (loco) na presença adicional de variância poligênica; todavia, o mesmo não ocorreu com o GP, que foi significativamente influenciado por efeitos poligênicos. Para as características GIM e ET, as médias marginais para as variâncias explicadas pelo GEP $(0,37$ e 3,44$)$ foram sensivelmente superiores às variâncias influenciadas pelos efeitos poligênicos do modelo $(0,11$ e 1,11), enquanto as variâncias residuais $(0,27$ e 6,03$)$ foram inferiores às estimadas quando se consideraram somente os efeitos poligênicos $(0,43$ e 7,51$)$ (Tabela 2; Figura 3). No entanto, ao estudar a característica GP, houve influência mais acentuada do efeito poligênico (1.900) e a influência do GEP somente foi superior quando se desprezou o efeito da dominância (3.080). As estimativas das herdabilidades para $\operatorname{GIM}(0,24)$ e 
Tabela 2 - Estimativas marginais a posteriori médias e respectivos desvios-padrão para as variâncias aditivas $\left(\sigma^{2}{ }_{a}\right)$, de dominância $\left(\sigma_{d}^{2}\right)$, do gene de efeito principal $\left(\sigma_{\text {GEP }}^{2}\right)$, poligênica $\left(\sigma_{P}^{2}\right)$ e residual $\left(\sigma^{2}{ }_{e}\right)$ e herdabilidade para o gene de efeito principal $\left(h^{2}\right.$ GEP $)$ e poligênica $\left(h^{2}{ }_{P}\right)$ para gordura intramuscular $(\mathrm{GIM})$, espessura de toucinho (ET) e ganho de peso (GP)

Table 2 - Estimated marginal posterior means and marginal posterior standard deviations for additive variance ( $\left.\sigma^{2}{ }_{a}\right)$, dominance variance $\left(\sigma^{2}{ }_{d}\right)$, major gene variance $\left(\sigma^{2}{ }_{G E P}\right)$, polygenic variance $\left(\sigma_{P}^{2}\right)$ and error variance $\left(\sigma^{2}{ }_{e}\right)$ and heritability of major gene $\left(h_{\text {GEP }}^{2}\right)$ and polygenic $\left(h^{2}\right)$ for intramuscular fat (GIM), backfat thickness $(E T)$ and weight gain (GP)

\begin{tabular}{|c|c|c|c|c|c|}
\hline \multirow{2}{*}{$\begin{array}{l}\text { Modelo } \\
\text { Model } \\
\text { Parâmetro } \\
\text { Parameter }\end{array}$} & \multicolumn{2}{|c|}{$\mathrm{MPI}+\mathrm{MPF}^{1}$} & \multirow[t]{2}{*}{$\mathrm{MPI}^{2}$} & \multicolumn{2}{|c|}{$\mathrm{MPF}^{3}$} \\
\hline & $\sigma_{\mathrm{a}}^{2}$ & $\sigma_{a}^{2}+\sigma_{d}^{2}$ & & $\sigma_{a}^{2}$ & $\sigma_{a}^{2}+\sigma_{d}^{2}$ \\
\hline \multicolumn{6}{|l|}{ GIM } \\
\hline $\begin{array}{l}\sigma^{2} \\
\sigma^{2}{ }^{\mathrm{a}} \\
\sigma^{2}{ }^{2} \\
\mathrm{~h}^{2}{ }^{\mathrm{GEP}} \\
\sigma^{2}{ }_{\mathrm{PEP}} \\
\mathrm{h}^{2}{ }_{\mathrm{P}} \\
\sigma_{\mathrm{e}}{ }_{\mathrm{P}}\end{array}$ & $\begin{array}{l}0,63(0,12) \\
0,63(0,12) \\
\mathbf{0 , 6 5}(\mathbf{0 , 0 5}) \\
0,03(0,03) \\
\mathbf{0 , 0 3}(\mathbf{0 , 0 3}) \\
0,31(0,03)\end{array}$ & $\begin{array}{l}0,19(0,09) \\
0,18(0,06) \\
0,37(0,12) \\
\mathbf{0 , 2 4}(\mathbf{0 , 0 9}) \\
0,11(0,04) \\
\mathbf{0 , 1 5}(\mathbf{0 , 0 6}) \\
0,27(0,03)\end{array}$ & $\begin{array}{l}0,26(0,08) \\
\mathbf{0 , 3 7}(\mathbf{0 , 1 0}) \\
0,43(0,06)\end{array}$ & $\begin{array}{l}0,67(0,13) \\
\mathbf{0 , 7 1}(\mathbf{0 , 0 6})\end{array}$ & $\begin{array}{l}0,31(0,10) \\
0,26(0,07) \\
0,57(0,13) \\
\mathbf{0 , 4 0}(\mathbf{0 , 0 7})\end{array}$ \\
\hline \multicolumn{6}{|l|}{$\overline{\mathrm{ET}}$} \\
\hline $\begin{array}{l}\sigma^{2} \\
\sigma^{2}{ }^{\mathrm{a}} \\
\sigma^{2} \\
\mathrm{~h}^{2}{ }_{\mathrm{GEP}} \\
\sigma^{2}{ }_{\mathrm{PEP}} \\
\mathrm{h}^{2}{ }_{\mathrm{P}} \\
\sigma_{\mathrm{e}}{ }^{2}\end{array}$ & $\begin{array}{l}2,95(1,53) \\
2,95(1,53) \\
\mathbf{0 , 2 8}(\mathbf{0 , 1 4}) \\
1,54(1,09) \\
\mathbf{0 , 0 9}(\mathbf{0 , 0 8}) \\
6,91(1,00)\end{array}$ & $\begin{array}{l}2,59(1,47) \\
0,85(0,58) \\
3,44(1,40) \\
\mathbf{0 , 2 4}(\mathbf{0 , 1 3}) \\
1,11(0,80) \\
\mathbf{0 , 1 0}(\mathbf{0 , 0 7}) \\
6,03(0,81)\end{array}$ & $\begin{array}{l}2,36(0,78) \\
\mathbf{0 , 2 4}(\mathbf{0 , 0 7}) \\
7,51(0,07)\end{array}$ & $\begin{array}{l}3,98(1,25) \\
\\
3,98(1,25) \\
\mathbf{0 , 3 9 ( 0 , 1 0 )}\end{array}$ & $\begin{array}{l}4,35(1,38) \\
1,55(0,90) \\
5,89(1,58) \\
\mathbf{0 , 3 9 ( 0 , 1 0 )}\end{array}$ \\
\hline \multicolumn{6}{|l|}{$\overline{\mathrm{GP}}$} \\
\hline $\begin{array}{l}\sigma^{2} \\
\sigma^{2}{ }^{\mathrm{d}} \\
\sigma^{2} \\
\mathrm{~h}^{2}{ }_{\mathrm{GEP}} \\
\sigma^{2}{ }_{\mathrm{GEP}} \\
\mathrm{h}^{2}{ }_{\mathrm{P}} \\
\sigma_{\mathrm{e}}^{2}{ }_{\mathrm{e}}\end{array}$ & $\begin{array}{l}3080(1460) \\
\mathbf{0 , 4 7}(\mathbf{0 , 1 1}) \\
1410(810) \\
\mathbf{0 , 2 3}(\mathbf{0 , 1 3}) \\
1750(570)\end{array}$ & $\begin{array}{l}110(260) \\
1340(750) \\
1450(800) \\
\mathbf{0 , 0 2}(\mathbf{0 , 0 4}) \\
1900(860) \\
\mathbf{0 , 4 1}(\mathbf{0 , 1 7}) \\
1260(600)\end{array}$ & $\begin{array}{l}1460(790) \\
\mathbf{0 , 3 7}(\mathbf{0 , 1 7}) \\
2410(560)\end{array}$ & $\begin{array}{l}4920(1530) \\
\mathbf{0 , 6 8}(\mathbf{0 , 1 1})\end{array}$ & $\begin{array}{l}310(260) \\
2150(820) \\
2460(870) \\
\mathbf{0 , 0 7}(\mathbf{0 , 0 6})\end{array}$ \\
\hline
\end{tabular}

1 - um loco; 2 - Modelo Poligênico Infinitesimal; 3 - Modelo Poligênico Finito (\#5 locos).

1 - one locus; 2 - Infinitesimal Polygenic Model; 3 - Finite Polygenic Model (\# 5 loci).

Tabela 3 - Distribuição a posteriori para número de GEP $\left[P\left(N_{G E P}\right)\right]$ e número esperado de GEP (NGEP), quando se ajustou o modelo poligênico finito, considerando cinco locos

Table 3 - Posteriori distribution of number of GEP $\left[P\left(N_{G E P}\right)\right]$, when fitted finite polygenic model, with five loci

\begin{tabular}{|c|c|c|c|c|c|c|c|}
\hline & \multicolumn{6}{|c|}{$\mathrm{P}\left(\mathrm{N}_{\mathrm{GEP}}\right)$} & \multirow[t]{2}{*}{ NGEP } \\
\hline & 0 & 1 & 2 & 3 & 4 & 5 & \\
\hline \multicolumn{8}{|l|}{$M P F-d=0$} \\
\hline GIM(\%) & 0,0000 & 0,2348 & 0,7408 & 0,0240 & 0,0004 & 0,0000 & 1,79 \\
\hline ET(mm) & 0,0000 & 0,8584 & 0,1369 & 0,0047 & 0,0000 & 0,0000 & 1,15 \\
\hline GP (g/dia) & 0,0000 & 0,0841 & 0,7969 & 0,1147 & 0,0043 & 0,0000 & 2,04 \\
\hline \multicolumn{8}{|l|}{$\overline{\mathrm{MPF}-\mathrm{d} \neq 0}$} \\
\hline GIM(\%) & 0,0000 & 0,0000 & 0,3728 & 0,5831 & 0,0392 & 0,0049 & 2,68 \\
\hline $\mathrm{ET}(\mathrm{mm})$ & 0,0000 & 0,0311 & 0,7784 & 0,1784 & 0,0152 & 0,0012 & 2,18 \\
\hline GP (g/dia) & 0,0000 & 0,0004 & 0,8848 & 0,1121 & 0,0027 & 0,0000 & 2,18 \\
\hline
\end{tabular}



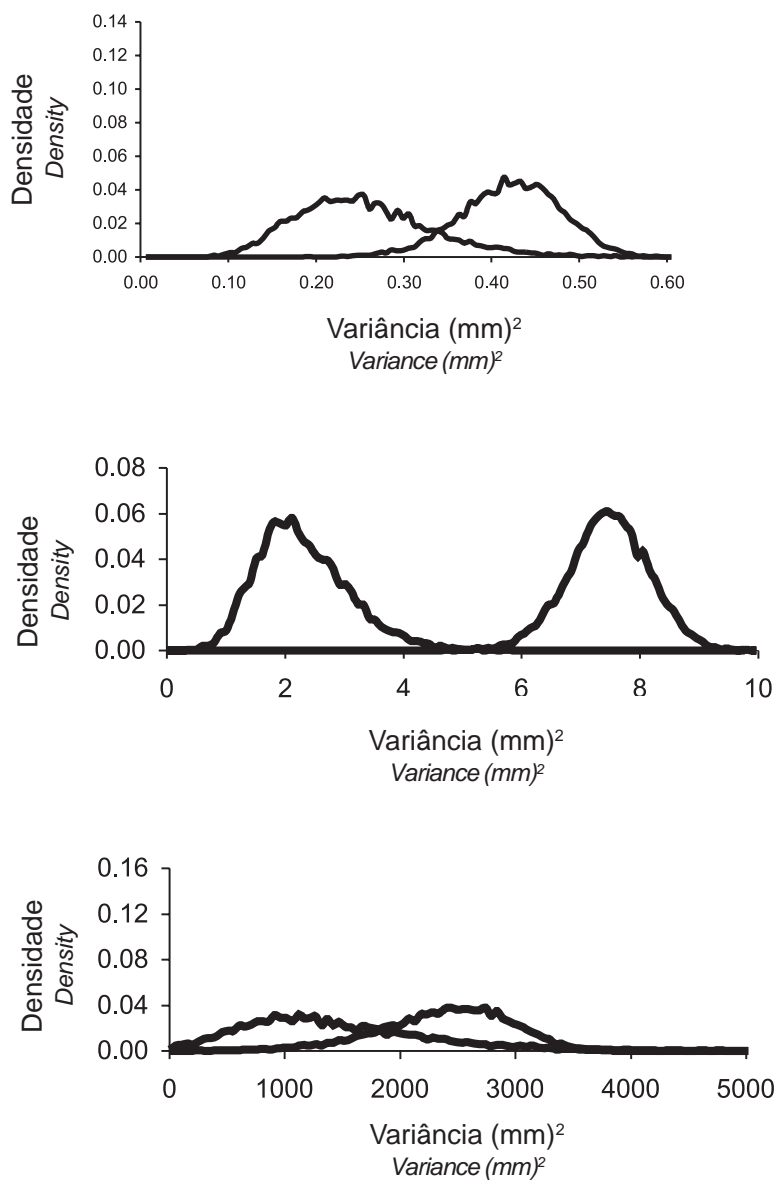
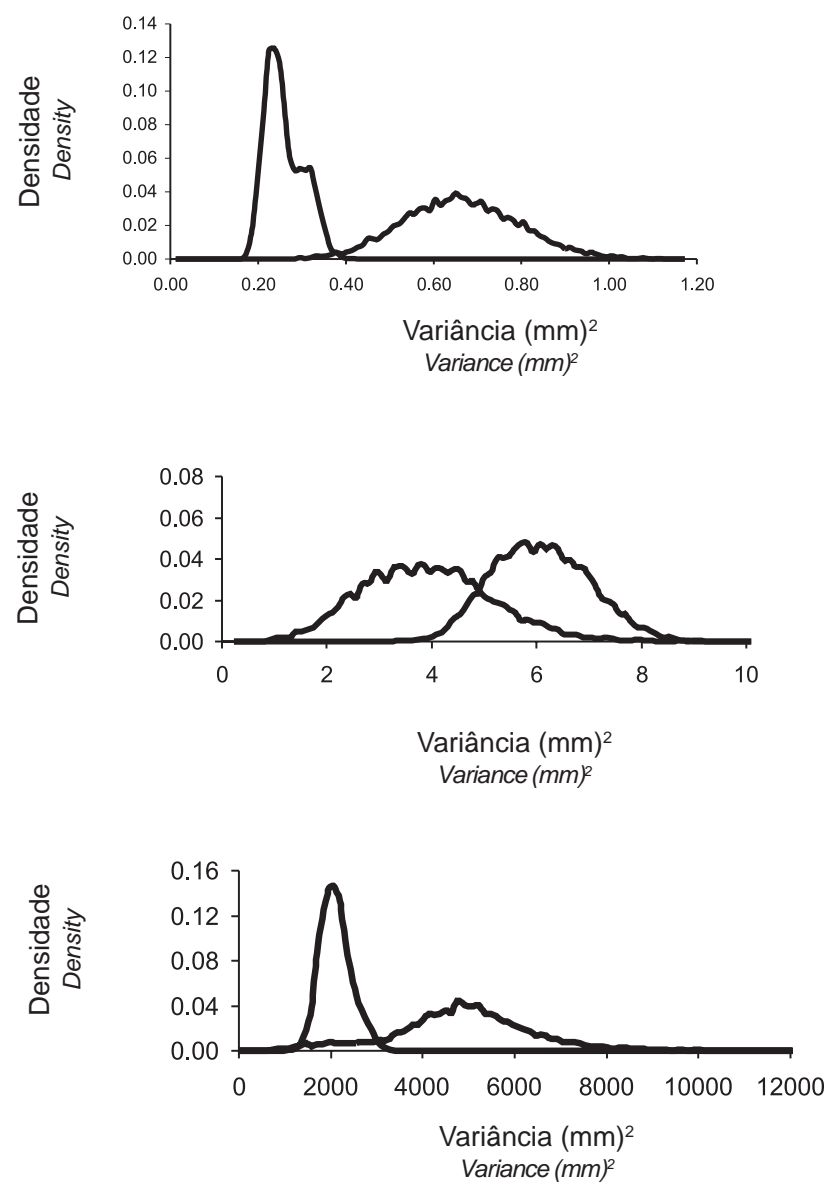

Figura 1 - Distribuição marginal a posteriori para variância poligênica $\left(\sigma^{2}\right.$ ) estimada no MPI, variância aditiva no MPF $\left(\sigma^{2}{ }_{a}\right)$ e respectivas variâncias residuais $\left(\sigma^{2}\right)$ para GIM, ET e GP.

Figure 1 - Marginal posteriori distributions of polygenic variances $\left(\sigma_{P}^{2}\right)$ in MPI, additive variance in MPF $\left(\sigma^{2}{ }_{a}\right)$ and error variance $\left(\sigma^{2}{ }_{e}\right)$ for GIM, ET and GP.

ET $(0,24)$ considerando-se um loco simples $\left(h^{2}{ }_{G E P}\right)$ foi maior que a apresentada pela parte poligênica $(0,15$ e 0,10 ) do modelo (Figura 4) e, para a GIM, quando se considerou o efeito da dominância, a soma das herdabilidades $\left(\mathrm{h}^{2}{ }_{\mathrm{GEP}} \mathrm{e} \mathrm{h}^{2}{ }_{\mathrm{P}}\right)$ foi próxima à estimada no modelo poligênico infinitesimal $(0,37)$. Comparando com a variância poligênica apresentada no MPI, pode-se constatar que apenas um GEP foi suficiente para explicar a variação ocorrida na característica $\mathrm{ET}$, considerando-se ou não o efeito da dominância. A variância genética apresentada pela característica GP, quando se combinou MPI e MPF e considerando-se o efeito de dominância, foi explicada praticamente pelos efeitos poligênicos, não tendo praticamente nenhuma influência do GEP.

Com o propósito de se tentar discernir entre os diferentes fatores genéticos que influenciam algumas características quantitativas de suínos, foram empregados diferentes modelos hierárquicos. Para o MPF e na combinação dos MPF e MPI, a variância genética para as características GIM e ET foi significativamente explicada por GEP (Tabela 2), o que pode ser confirmado pela distribuição de probabilidade a posteriori da variância genética e/ou das herdabilidades (Figuras 1 a 4). Para as características estudadas, principalmente ET, a probabilidade $a$ posteriori de se ter valores da variância genética iguais a zero ou muito baixos para MPF e para a combinação dos modelos foi baixa (Figuras 3 e 4). Segundo Bink et al. (2002a), somente se pode postular a possibilidade de se ter GEP quando não se verifica grande quantidade de variância genética (ou herdabilidade) próxima ou igual a zero. 

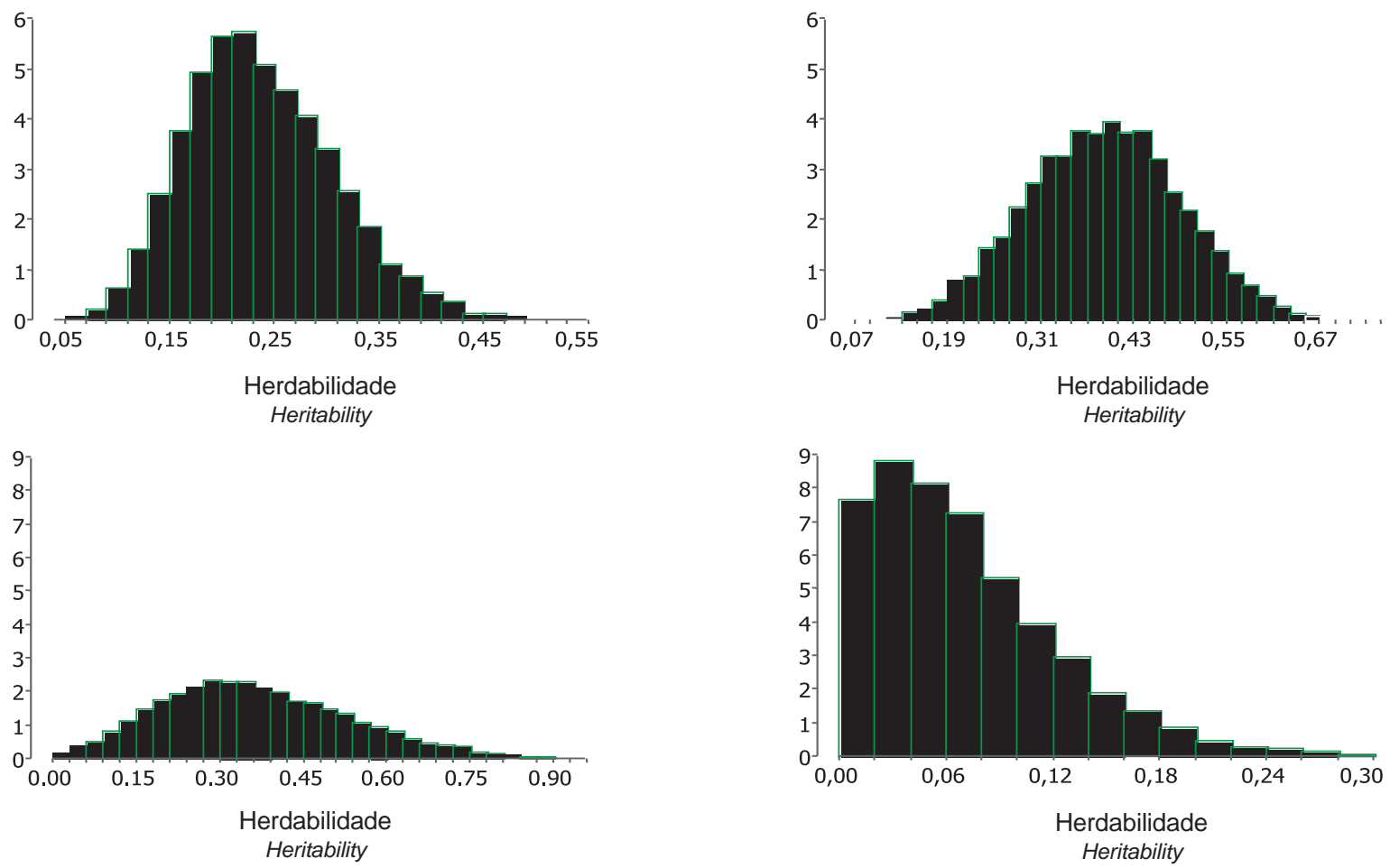

Figura 2 - Distribuições marginais a posteriori para as herdabilidades estimadas nos modelos poligênico infinitesimal (à esquerda) e finito (à direita) para as características GIM, ET e GP.

Figure 2 - Marginal posteriori distributions for estimated heritability in infinitesimal polygenic model (left) and finite polygenic model (right) for GIM, ET and GP.

Tabela 4 - Estimativa média a posteriori da variância aditiva $\left(\sigma^{2}{ }_{a}\right)$ e de dominância $\left(\sigma^{2}{ }_{d}\right)$, para os cincos locos considerados no modelo poligênico finito

Table 4 - Estimated marginal posterior means of additive variance $\left(\sigma^{2}{ }_{a}\right)$ and dominance variance $\left(\sigma^{2}{ }_{d}\right)$, when fitted finite polygenic model, with five loci

\begin{tabular}{|c|c|c|c|c|c|c|c|c|c|c|}
\hline \multirow[b]{2}{*}{$\begin{array}{l}\text { Locos } \\
\text { Loci }\end{array}$} & \multicolumn{5}{|c|}{$\begin{array}{l}\text { Variância aditiva }\left(\sigma^{2}{ }_{a}\right) \\
\text { Additive variance }\left(\sigma_{a}^{2}\right)\end{array}$} & \multicolumn{5}{|c|}{$\begin{array}{c}\text { Variância dominância }\left(\sigma_{\mathrm{d}}^{2}\right) \\
\text { Dominance variance }\left(\sigma_{d}^{2}\right)\end{array}$} \\
\hline & 1 & 2 & 3 & 4 & 5 & 1 & 2 & 3 & 4 & 5 \\
\hline \multicolumn{11}{|c|}{$M P F-d=0$} \\
\hline GIM & 0,57 & 0,13 & 0,01 & 0,01 & & & & & & \\
\hline ET & 3,48 & 3,51 & 1,94 & & & & & & & \\
\hline \multicolumn{11}{|c|}{$M P F-d \neq 0$} \\
\hline GIM & 0,16 & 0,10 & 0,09 & 0,08 & 0,08 & 0,19 & 0,05 & 0,04 & 0,05 & 0,04 \\
\hline ET & 1,58 & 2,35 & 1,86 & 2,37 & 0,46 & 0,78 & 0,62 & 0,77 & 0,66 & 0,21 \\
\hline GP & 50 & 240 & 230 & 050 & & 1330 & 730 & 730 & 090 & \\
\hline
\end{tabular}



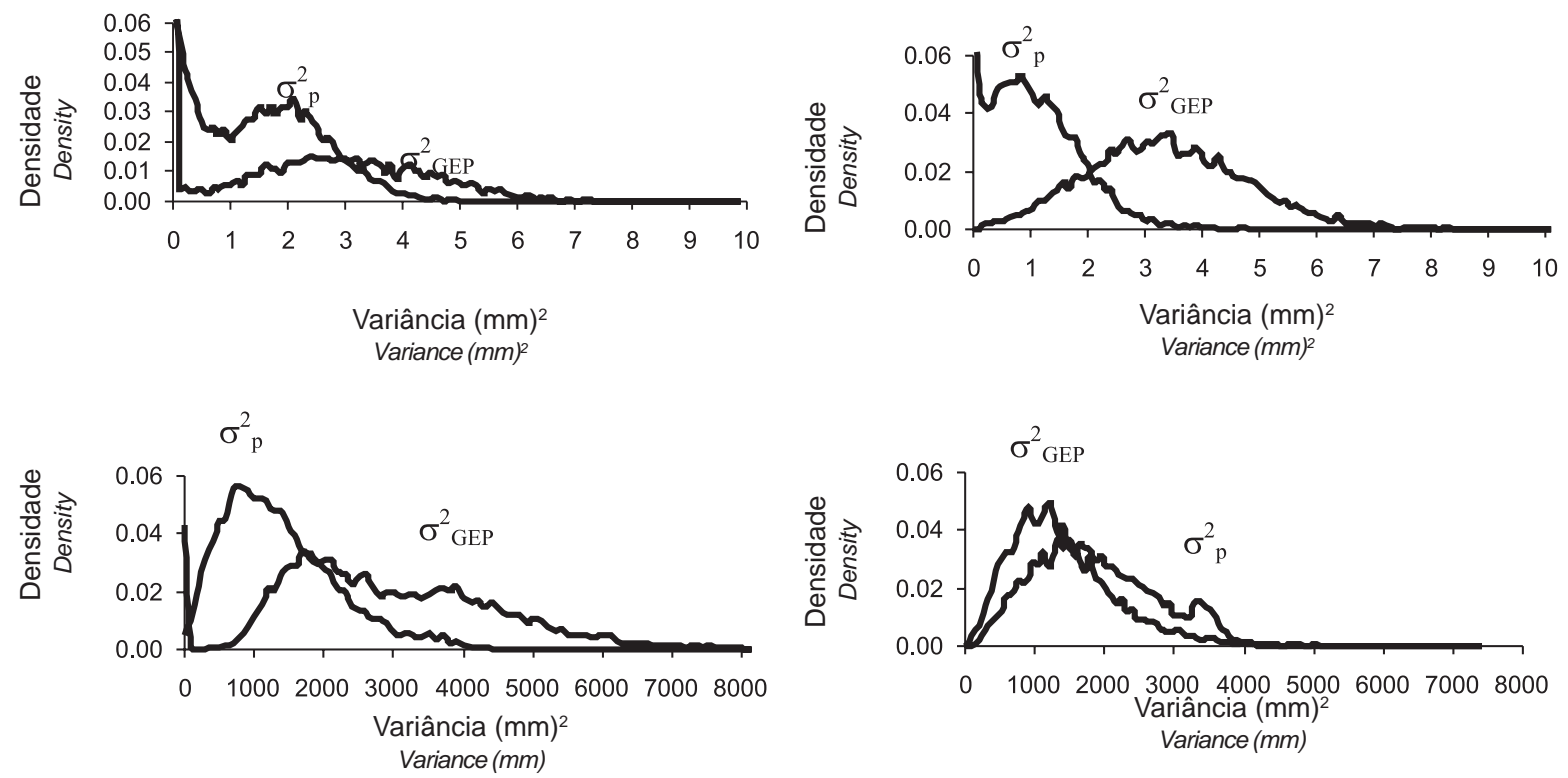

Figura 3 - Distribuições marginais a posteriori para as variâncias genéticas poligênica $\left(\sigma^{2}\right)$ e do gene de efeito principal $\left(\sigma^{2}\right.$ GEP $)$ com $d=0$ (a esquerda) e $d \neq 0$ (a direita) quando combinados os MPI e MPF para as características GIM, ET e GP.

Figure 3 - Marginal posteriori distributions for polygenic variance $\left(\sigma_{P}^{2}\right)$ and major gene variance $\left(\sigma_{G E P}^{2}\right)$ with $d=0$ (left) and $d \neq 0$ (right) when fitted the combination of models polygenic infinitesimal and finite for GIM, ET and GP.
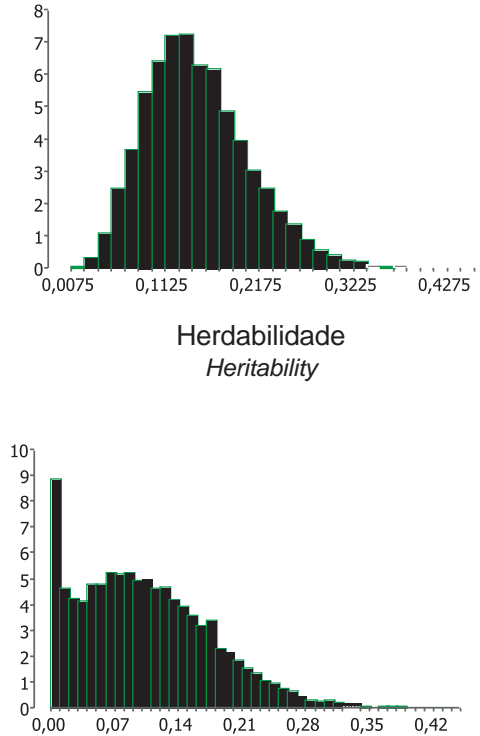

Herdabilidade Heritability

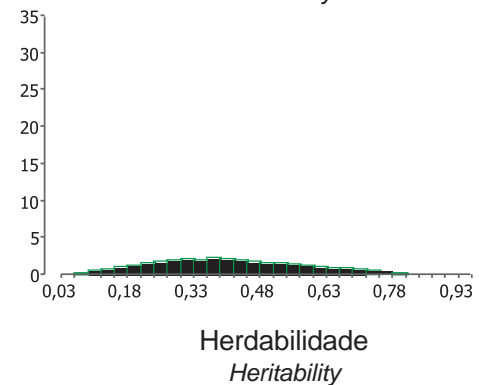

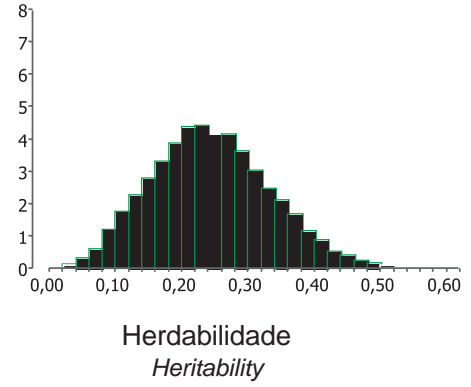

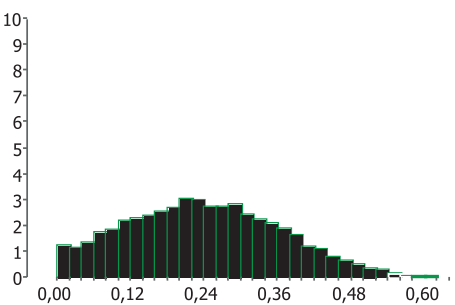

Herdabilidade Heritability

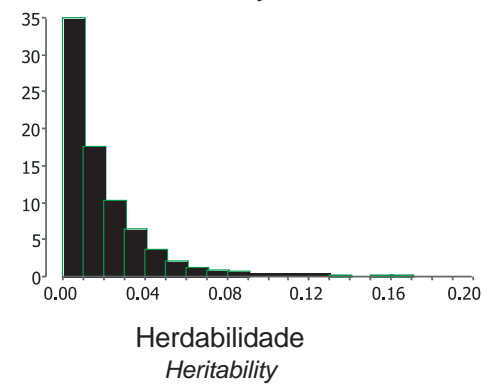

Figura 4 - Distribuições marginais a posteriori para as herdabilidades estimadas para as características GIM, ET e GP, considerando-se os efeitos genéticos poligênicos (à esquerda) e de GEP (à direita) quando combinados os MPI e MPF.

Figure 4 - Marginal posteriori distributions for estimated heritability for GIM, ET and GP considered polygenic variance (left) and major gene variance (right) when fitted the combination of MPI and MPF. 


\section{Conclusões}

A metodologia utilizada (Bayesiana) neste estudo foi eficiente na análise do pedigree e dos fenótipos de suínos cruzados, identificando os diferentes componentes de variação genética e estimando suas magnitudes.

Cinco GEP (efeitos aditivos e de dominância ajustados) foram identificados, três para gordura intramuscular (GIM) e dois para espessura de toucinho (ET). Para ET, os GEP explicaram a maior parte da variância genética, enquanto, para GIM, os GEP reduziram significativamente a variância poligênica. Para a característica ganho de peso (GP), um GEP foi observado, mas não se observou alteração expressiva da variação poligênica, possivelmente em razão da complexidade do controle genético da característica.

Com base nestes resultados, conclui-se que os dados de cruzamento podem ser úteis para a localização de genes afetando GIM, ET e, possivelmente, GP. Os resultados da análise de ligação marcador genético e gene afetando estas características serão importante ferramenta na determinação dos genes verdadeiramente responsáveis pela variação das mesmas.

\section{Literatura Citada}

ALLAIRE, F.R.; HENDERSON, C.R. Specific combining abilities among dairy sires. Journal of Dairy Science, v.48, p.1096-1100, 1965.

BINK, M.C.A.M.; UIMARI, P.; SILLANPÄÄ, M.J. et al. Multiple QTL mapping in related plant populations via a pedigree analysis approach. Theoretical and Applied Genetics, v.104, p.751-762, 2002a.

BINK, M.C.A.M.; UIMARI, P.; TE PAS, M.F.W. et al. Statistical inference on genetic response in selection lines using Bivariate finite polygenic and QTL models. In: WORLD CONGRESS ON GENETICS APPLIED TO LIVESTOCK PRODUCTION, 7., 2002, Montpellier. Proceedings... Montpellier: 2002b. v.32, p.729-732.

DE KONING, D.; RATTINK, A.P.; HARLIZIUS, B. et al. Detection and characterization of quantitative trait loci for meat quality traits in pigs. Journal of Animal Science, v.79, p.2812-2819, 2001.

DU, F.X.; HOESCHELE, I.; GAGE-LAHTI, K.M. Estimation of additive and dominance variance components in finite polygenic models and complex pedigrees. Genetics Review, v.74, p.179-187, 1999.

FALCONER, D.S.; MACKAY, T.F.C. Introduction to quantitative genetics. 4.ed. New York: Harlow, Essex: Addison Wesley Logman, 1996. 464p.

FERNANDO, R.L.; STRICKER, C.; ELSTON, R.L. The finite polygenic mixed model an alternative formulation for the mixed model of inheritance. Theoretical and Applied Genetics, v.88, p.573-580, 1994.

FUERST, C.; SOELKNER, J. Additive and nonadditive genetic variance of milk yield, fertility, and life time performance traits of dairy cattle. Journal of Dairy Science, v.77, p.1114-1125, 1994.

GREEN, P.J. Reversible jumping Markov chain Monte Carlo computation and Bayesian model determination. Biometrics, v.82, p.711-732, 1995.

HAY, G.M.; WHITE, J.M; VINSON, W.E. et al. Components of genetic variation for descriptive type traits of Holsteins. Journal of Dairy Science, v.66, p.1962-1966, 1983.

HOESCHELE, I. Additive and nonadditive genetic variance in female fertility of Holsteins. Journal of Dairy Science, v.74, p.1743-1752, 1991.

HOESCHELE, I; UIMARI, P.; GRIGNOLA, F.E. et al. Advances in statistical methods to map quantitative trait loci in outbreed populations. Genetics, v.147, p.1445-1457, 1997.

HOVENIER, R.; KANIS, E.; Van ASSELDONK, T. et al. Breeding for pig meat quality in halothane-negative populations - a review. Pig News and Information, v.14, p.17-25, 1993.

JANSS, L.L.G; THOMPSON, R.; Van ARENDONK, J.A.M. Application of Gibbs sampling for inference in a mixed major gene-polygenic inheritance model in animal populations. Theoretical and Applied Genetics, v.91, p.1137-1147, 1995.

JANSS, L.L.G.; VAN ARENDONK, J.A.M.; BRASCAMP, E.W. Bayesian statistical analyses for presence of single genes affecting meat quality traits in a crossed pig population. Genetics, v.145, p.395-408, 1997.

LANGE, K.; MATTHYSSE, S. Simulation of pedigree genotypes by random walks. The American Journal of Human Genetics, v.45, p.959-970, 1989.

PONG-WONG, R.; SHAW, F.; WOOLLIAMS, J.A. Estimation of dominance variation using a finite-locus model. In: CONGRESS ON THE QUANTITATIVE GENETICS OF LIVESTOCK, 6., 1998, Armidale. Proceedings... Armidale: 1998. v.26, p.41-44.

PONG-WONG, R.; HALEY, C.S.; WOOLLIAMS, J.A. Behavior of the additive finite locus model. Journal Genetics Selection Evolution, v.31, p.193-211, 1999.

SILLANPÄÄ, M.J.; ARJAS, E. Bayesian mapping of multiple quantitative trait loci from incomplete inbred line cross data. Genetics, v.148, p.1373-1388, 1998.

SOBEL, E.; LANGE, K. Descent graphs in pedigree analysis: applications to haplotyping, location scores, and markersharing statistics. The American Journal of Human Genetics, v.58, p.1323-1337, 1996.

SZYDA, J.; LIU, Z.; GRINDFLEK, E. et al. Application of a mixed inheritance model to the detection of quantitative trait loci in swine. Journal of Applied Genetics, v.43, p.69-83, 2002.

THOMPSON, E.A.; SKOLMICK, M.H. Likelikoods on complex pedigrees for quantitative traits. In: INTERNATIONAL CONFERENCE ON QUANTITATIVE GENETICS, 1977, Ames. Proceedings... Ames: Iowa State University Press, 1977. p.815-818.

THOMPSON, E.A. Monte Carlo likelihood in genetic mapping. Statistics Science, v.9, p.903-908, 1994.

WARRIS, P.D.; BROWN, S.N.; FRANKLIN, J.G. et al. The thickness and quality of backfat in various pig breeds and their relationship to intramuscular fat and the setting of the joints from the carcasses. Meat Science, v.28, p.21-29, 1990. 